\title{
Acute hemichorea as unusual first multiple sclerosis presentation
}

Giada Giovannini, MD; Francesco Cavallieri, MD; Stefano Meletti, MD, PhD; Annalisa Chiari, MD; Jessica Mandrioli, MD; Diana Ferraro, MD, PhD; Franco Valzania, MD

$\mathrm{P}$ atient 1 was a 39-year-old woman with an unremarkable medical history who developed acute involuntary right arm and leg movements. Neurologic examination revealed moderate dysarthria and subcontinuous, choreic movements in her right limbs, prevailing in the arm, which worsened during postural tasks. She occasionally had ballistic movements in her right limbs and abnormal dystonic postures. Continuous peribuccal and tongue involuntary movements were noted. Moreover, bilateral upper limb ataxia, gait and trunk ataxia, and brisk right tendon reflexes were found. There was no strength or sensory loss (video 1 at Neurology.org/cp). Brain MRI revealed a tumefactive, T2/fluidattenuated inversion recovery (FLAIR) hyperintense, T1 hypointense contrast-enhancing demyelinating lesion in the left cerebral peduncle, extending to the substantia nigra and subthalamic nucleus (STN) (figure, A-C). Multiple hyperintense T2/FLAIR, T1 hypointense, non-contrast-enhancing demyelinating lesions in the hemispheric and periventricular deep white matter, brainstem, and cerebellar hemispheres were also found. All serologic tests were within normal limits. Isoelectric focusing (IEF) revealed 9 CSF oligoclonal bands (OCBs). A diagnosis of multiple sclerosis (MS) was made and the patient was treated with high-dose methylprednisolone with improvement of symptoms.

Patient 2 was an 18-year-old man with an unremarkable medical history who developed acute involuntary left arm and leg movements, dysarthric speech, and mental slowing. Neurologic examination showed intermittent choreic and occasional choreoathetoid movements associated with a dystonic posture affecting predominantly the left forearm, hand, or fingers (video 2). There was no strength, coordination, or sensory loss. Brain MRI revealed a right subthalamic T2/FLAIR hyperintense, T1 hypointense, contrast-enhancing demyelinating lesion extending to the homolateral cerebral peduncle (figure, D-F). Multiple T2/FLAIR hyperintense, T1 hypointense non-contrast-enhancing demyelinating lesions involved the left part of the ponsmesencephalic passage, the homolateral superior cerebellar peduncle, and the corpus callosum. All serologic and urinary tests, including ceruloplasmin and copper levels, were within normal limits. IEF revealed 5 CSF OCBs. A diagnosis of MS was made and the patient was treated with high-dose methylprednisolone. The involuntary movements completely resolved in 6 days. One month later, the patient developed left facial hemispasm. Brain MRI did not reveal new lesions. He was newly treated with steroids. Clonazepam and botulinum toxin injection in the left orbital muscle were also administered, leading to a complete resolution of symptoms.

\section{DISCUSSION}

Hyperkinetic movement disorders, in particular chorea and ballism, have been rarely reported in patients with MS and almost never represent the first clinical manifestation. ${ }^{1}$ The few cases

\section{Practical Implications}

Acute onset of unilateral dyskinesia, similar to those induced by levodopa in advanced Parkinson disease, should suggest a functional inhibition of the subthalamic nucleus. Aside from common cases induced by metabolic or vascular lesions, this clinical picture in young patients could suggest an unusual onset of a CNS demyelinating disease.

Videos

Neurology.org/cp

Department of Neuroscience, S. Agostino-Estense Hospital and University of Modena and Reggio Emilia, Modena, Italy.

Funding information and disclosures are provided at the end of the article. Full disclosure form information provided by the authors is available with the full text of this article at Neurology.org/cp.

Correspondence to: giovannini.giada@gmail.com 

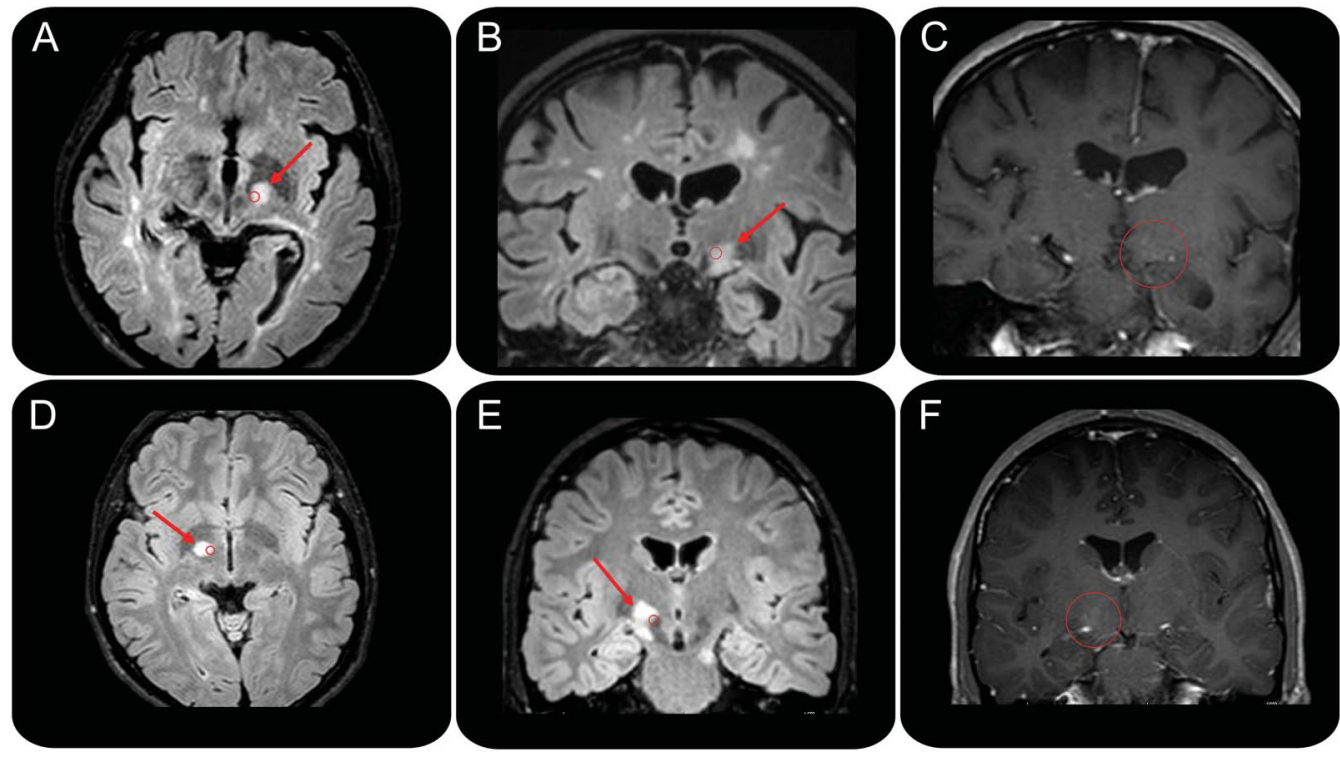

Three tesla brain MRI obtained before and after IV gadolinium injection. Fluid-attenuated inversion recovery sequences: 2 -mm slice thickness, $1.2 \times 1.2 \mathrm{~mm}^{2}$ in-plane resolution. T1-weighted postcontrast sequences: 4-mm slice thickness, $0.85 \times 1.06 \mathrm{~mm}^{2}$ in-plane resolution. Patient 1: axial fluid-attenuated inversion recovery brain MRI shows a tumefactive, hyperintense demyelinating lesion (red arrow) in the left subthalamic region (red circle) in addition to multiple demyelinating lesions in the hemispheric deep white matter. (A) Coronal fluid-attenuated inversion recovery brain MRI shows the same signal abnormalities (red arrow) in the left cerebral peduncle, extending to the substantia nigra and subthalamic nucleus (red circle) with bilateral hyperintense demyelinating lesions in the periventricular white matter (B). Coronal T1-weighted postcontrast brain MRI of the same anatomic area of image $B$ demonstrates gadolinium enhancement of the subthalamic lesion (red circle) (C). Patient 2: axial fluid-attenuated inversion recovery brain MRI reveals a wide hyperintense demyelinating lesion (red arrow) in the right upper subthalamic region (red circle) (D). Coronal fluid-attenuated inversion recovery brain MRI shows hyperintense signal abnormalities (red arrow) in right cerebral pedunculus, homolateral substantia nigra, subthalamic nucleus (red circle), and left part of the pons-mesencephalic passage (E). Coronal T1-weighted postcontrast brain MRI demonstrates gadolinium enhancement of the subthalamic lesion (red circle) (F).

reported in literature had basal ganglia (mainly striatum) demyelinating plaques. ${ }^{1}$ The occurrence of demyelinating lesions in the basal ganglia is explained by the presence of myelinated fibers within the subcortical gray nuclei. ${ }^{2}$ Historically, contralateral hemichorea has been described in relation to subthalamic or thalamic lesions due to various causes. ${ }^{3}$ In our patients, the active demyelinating lesions are localized almost exactly in the same regions as those identified by Lee and Marsden ${ }^{3}$ as being involved in the development of hemichorea. These observations, and the clinical improvement following steroid treatment, suggest a direct relationship between the occurrence of the demyelinating lesions and the onset of involuntary movements.

Since the STN is somewhat difficult to precisely localize using typical T1-and T2-weighted imaging, special contrast sequences such as susceptibility-weighted imaging have been developed. These sequences were not performed in our cases; this could have identified the precise localization of the STN. Thalamic lesions can cause a variety of movement disorders such as dystonia, tremor, asterixis, pseudochoreoathetosis (a movement disorder resulting from lesions involving sensory areas in the cortex, spinal cord, dorsal root ganglions, or thalamus), or hemichorea. ${ }^{4}$ In particular, a small thalamic lesion can cause either typical pseudochoreoathetotic finger movements, or variable, jerky chorea-like movements, worsened by eye closure. ${ }^{4}$ Unlike in pseudochoreoathetosis cases, no sensory deficits were detected in our patients. Choreoathetosis, with different degrees of dystonia, represents the clinical manifestation of peak-dose levodopa-induced dyskinesia in advanced Parkinson disease (PD). ${ }^{5}$ Drug-induced dyskinesias 
are possibly associated with STN hypoactivity, as suggested by the occurrence of dyskinesias in MPTP monkeys and by the occurrence of hemichorea-hemiballism in around 15\% of patients with PD after subthalamotomy or during deep brain stimulation..$^{5-7}$ In all these situations, there is a subthalamic hypoactivity, either transient or permanent, caused by pharmacologic, electrical, or surgical intervention. ${ }^{7}$

The acute onset of unilateral dyskinesias with semiology ranging from choreoathetosis to ballism or dystonia, similar to those induced by levodopa, should suggest a functional inhibition of the STN, with imbalance of motor control operated by the basal ganglia network. Aside from common cases induced by metabolic or vascular lesions, in young patients, this clinical picture could suggest an unusual onset of a demyelinating disease.

\section{REFERENCES}

1. Mehanna R, Jankovic J. Movement disorders in multiple sclerosis and other demyelinating diseases. J Neurol Sci 2013;328:1-8.

2. Minagar A, Sheremata WA, Weiner WJ. Transient movement disorders and multiple sclerosis. Parkinsonism Relat Disord 2002;9:111-113.

3. Lee MS, Marsden CD. Movement disorders following lesions of the thalamus or subthalamic region. Mov Disord 1994;9:493-507.

4. Kim JW, Kim SH, Cha JK. Pseudochoreoathetosis in four patients with hypesthetic ataxic hemiparesis in a thalamic lesion. J Neurol 1999;246:1075-1079.

5. Guridi J, González-Redondo R, Obeso JA. Clinical features, pathophysiology, and treatment of levodopa-induced dyskinesias in Parkinson's disease. Parkinsons Dis 2012;2012:943159.

6. Moyer JT, Danish SF. Stimulation-induced dyskinesias inform basal ganglia models and the mechanisms of deep brain stimulation. J Neurosci 2007;27:1799-1800.

7. Guridi J, Obeso JA. Letter to the editor: Parkinson's disease and early subthalamotomy. J Neurosurg 2015;122:980-981.

Received February 12, 2016. Accepted in final form April 12, 2016.

\section{AUTHOR CONTRIBUTIONS}

G. Giovannini: study concept and design, acquisition of data, analysis and interpretation of data, drafting the manuscript. F. Cavallieri: study concept and design, acquisition of data, analysis and interpretation of data, study supervision. S. Meletti: study concept and design, acquisition of data, analysis and interpretation of data, study supervision. A. Chiari: study concept and design, acquisition of data, analysis and interpretation of data, drafting the manuscript. J. Mandrioli: study concept and design, acquisition of data, analysis and interpretation of data. D. Ferraro: acquisition of data, analysis and interpretation of data, drafting the manuscript. F. Valzania: study concept and design, acquisition of data, analysis and interpretation of data, drafting the manuscript, study supervision.

\section{STUDY FUNDING}

No targeted funding reported.

\section{DISCLOSURES}

G. Giovannini and F. Cavallieri report no disclosures. S. Meletti has served on scientific advisory boards and as a consultant for UCB and EISAI and has received research support from the Ministry of Health and CaRisMo Foundation. A. Chiari reports no disclosures. J. Mandrioli has received funding for travel to attend congresses from Biogen Idec, Aventis Pharma, MedicAir, and Fondazione Italiana di Ricerca per la SLA (AISLA); serves on the editorial board of BioMed Research International: Neuroscience; and receives research support from ABScience, Emilia Romagna Region Health Authority, Programma di Ricerca Regione-Università 2010-2012, area 2, Ricerca per il Governo Clinico, and AISLA. D. Ferraro has received funding for travel and speaker honoraria from Merck Serono, TEVA Pharmaceutical Industries, Novartis, and Biogen Idec. F. Valzania reports no disclosures. Full disclosure form information provided by the authors is available with the full text of this article at Neurology.org/cp. 